ITC 2/48

Journal of Information Technology and Control

Vol. 48/No. 2/2019

pp. 268-277

DOI 10.5755/j01.itc.48.2.20406
Linear Data-Model Based Adaptive ILC for Freeway Ramp Metering Without Identical Conditions on Initial States and Reference Trajectory

\title{
Linear Data-Model Based Adaptive ILC for Freeway Ramp Metering Without Identical Conditions on Initial States and Reference Trajectory
}

\section{Hao Liang}

Engineering Institute, Yantai Nanshan University, No 12, University road, East Sea tourism resort, Longkou, Yantai, China

\section{$\mathrm{Na}$ Lin}

School of Automation \& Electronic Engineering, Qingdao University of Science \& Technology, No 99, Songling Road, Qingdao, China

\section{Ronghu Chi}

Engineering Institute, Yantai Nanshan University, No 12, University road, East Sea tourism resort, Longkou, Yantai, China;

School of Automation \& Electronic Engineering, Qingdao University of Science \& Technology, No 99, Songling Road, Qingdao, China

\section{Yumei Sun}

Engineering Institute, Yantai Nanshan University, No 12, University road, East Sea tourism resort, Longkou, Yantai, China 
Although freeway traffic system is conducted with a repeatable pattern day-to-day, the initial volume/or speed and the desired density of the traffic flow may vary with days due to the external disturbances. In this paper, a new linear data-model based adaptive iterative learning control (LDM-AILC) is proposed to address ramp metering in a macroscopic level freeway environment. A linear data-model is developed for the nonlinear macroscopic traffic flow model by introducing an equivalent dynamical linearization approach in the time domain. Then the LDM-AILC is designed with a feedback control law and a parameter updating law. The proposed scheme is data-driven intrinsically, where only the input and output data are required for the controller design and analysis. The convergence is shown by rigorous analysis without any identical conditions exposed on both the initial state and the reference trajectory. Extensive simulation results are provided to verify the effectiveness of the proposed LDM-AILC.

KEYWORDS: Adaptive ILC, Linear data-model, Traffic control, Ramp metering, Random initial conditions, Iteration-varying target trajectory.

\section{Introduction}

Freeway traffic control $[1,20,28]$ is an important area in the field of traffic engineering and intelligent transportation systems. Frequent congestions on freeway during rush hours deteriorate traffic conditions. The most common reasons causing freeway congestion include traffic demand being greater than capacity, as well as traffic accidents, road works and weather [28]. For better utilization of freeway capacity, ramp metering $[1,13,15,17,27]$ is a commonly implemented strategy. The purpose of ramp metering is to regulate the amount of traffic entering a given freeway at its entry ramps to maximize throughput by maintaining a desired (or optimal) occupancy on the downstream mainline freeway. Ramp metering is implemented by means of traffic lights in practice, which is used to meter the number of entering vehicles.

Based on the model extracted from these data, local and coordinated feedback (ALINEA) ramp metering strategy has been implemented [1]. It has been shown to be a remarkably simple, highly efficient and easily implemented ramp metering application. However it is difficult to calibrate the key model parameters critical to the performance, because model parameters vary with geometry of freeway road conditions and environmental factors, e.g. rain and frog. In addition, the freeway traffic flow system is of strong nonlinearities, coupling, and uncertain and thus its accurate model is hardly available in practice. Therefore, the common model-based control approaches [13, 15, 26-27] will encounter many unexpected difficulties when applied to freeway traffic control problems.

On the other hand, the macroscopic traffic flow patterns are in general repeated every day [11]. For in- stance, the traffic flow will start from a very low level at midnight, and increase gradually up to the first peak of morning rush hour, which is often from 7-9 AM, and the second one from 5-7 PM. Congestions typically start at the same location every day. Note that iterative learning control (ILC) $[8,10-12,16,25]$ is very suitable for a repetitive process. Based on this observation, some ILC based ramp metering strategies have been proposed for the freeway density control $[10-12,25,28]$ in daily or weekly basis. In [11], only basic ILC based ramp metering and speed control are discussed. In [10], the learning mechanism combined with a pure error feedback in a complementary manner is studied and the simulation results have shown its superiority to the ILC based only. In [12], the input saturation was explored by using ILC based ramp metering strategy and the complementary ramp metering combining ILC and ALINEA, respectively. In $[25,28]$, the effectiveness of the ramp metering based on ILC was further evaluated.

Note that all the above ILC approaches [10-12, 25, 28] for ramp metering are based on the contraction mapping and fixed-point theory to design linear iterative learning algorithm, which causes two major limitations when used in practice. The first one is that the transient performance of the system output along the iteration axis becomes poor sometimes because the convergence property of tracking error is achieved in the sense of $\lambda$ norm. The second one is that the identical conditions of initial states and target references must be matched to achieve a perfect tracking.

Actually, traffic control with strict day-to-day repeatability is the most ideal scenario. It is more 
practical that the initial values of traffic density and average speed of the vehicles, as well as the tracking target are varying day-to-day due to the variations of freeway road conditions and environmental factors, e.g. rain and frog. Therefore, it is certain interesting to explore the ILC approach for the freeway traffic system without a strictly repeatable pattern with random initial conditions and iteration-varying desired density trajectory.

Adaptive iterative learning control (AILC) scheme $[4,6-7,18,23-24]$ was widely studied with many successful applications. In general, the most attractive advantage of the AILC is its capability of dealing with the problems of iteration-varying reference trajectories, bounded but possibly large random initial resetting error and disturbance. However, most of the existing AILCs [4, 6-7, 23-25] depend on the fact that the unknown parameters are linearly parameterized with known nonlinear functions, thus cannot been utilized to the freeway traffic control problems due to the strong nonlinearity of the macroscopic traffic flow model.

Recently, neural networks or fuzzy systems have been applied to AILC in many works to deal with the problem that the plant nonlinearities cannot be linearly parameterizable such as those in [2, 3, 5, 19, 21-22]. However, it is often difficult to determine the fuzzy rule base and membership functions and to train the neural network with massive operation data. More recently, a data-driven model-free adaptive ILC [6, 14] was proposed for a class of nonlinear discretetime systems by introducing a dynamical linearization approach in the iteration domain. It achieves a perfect tracking under random initial conditions without requiring any external testing signals or any training process. But, the tracking target trajectory must be repeatable strictly.

Motivated by the above discussion, an equivalent dynamical linearization approach is proposed for freeway traffic system by introduce a new concept of pseudo partial derivative (PPD) [9]. Then, a new linear data-model based adaptive ILC (LDM-AILC) is proposed for the freeway density control via ramp metering. Both theoretical analysis and simulations results confirm the effectiveness of the proposed approach.

The main contributions of the proposed LDM-AILC are that: (a) it is capable of dealing with nonlinear systems without known linear parametric structures and thus it is a data-driven control approach where the controller design and analysis just depends on the I/O data. (b) It can deal with the iteration-varying uncertainties, such as random initial states and iteration-varying tracking targets and achieves a perfect tracking performance. In a sum, the proposed LDM-AILC is suitable in practice for a complicated freeway traffic system with higher orders and strong nonlinearities and non-repetitive changes.

The rest of this paper is organized as follows. Section 2 shows a linear data-model of the macroscopic traffic flow model. Section 3 presents the LDM-AILC approach with rigorous analysis. Section 4 provides the simulation results to verify the effectiveness of the proposed approach. Section 5 concludes this work.

\section{Problem Formulation}

\subsection{Nonlinear Macroscopic Traffic Flow Model}

As shown in Fig.1, a single freeway is considered with one on-ramp and one off-ramp on each section. Its spatially discretized traffic flow model is expressed mathematically by the following equations [17].

$$
\begin{aligned}
\rho_{i}(t+1) & =\rho_{i}(t)+\frac{h}{L_{i}}\left[q_{i-1}(t)-q_{i}(t)+r_{i}(t)-s_{i}(t)\right], \\
q_{i}(t)= & \rho_{i}(t) v_{i}(t), \\
v_{i}(t+1) & =v_{i}(t)+\frac{h}{\tau}\left[V\left(\rho_{i}(t)-v_{i}(t)\right]\right. \\
+ & \frac{h}{L_{i}} v_{i}(t)\left[v_{i-1}(t)-v_{i}(t)\right]-\frac{v h}{\tau L_{i}} \frac{\left[\rho_{i+1}(t)-\rho_{i}(t)\right]}{\left[\rho_{i}(t)+\kappa\right]},
\end{aligned}
$$

\section{Figure1}

Sections on a freeway with on/off ramp

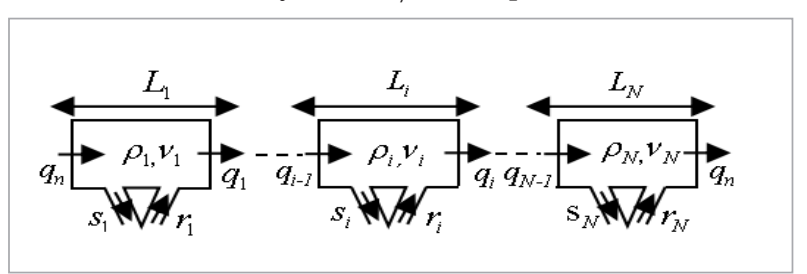




$$
V\left(\rho_{i}(t)=v_{\text {free }}\left(1-\left[\frac{\rho_{i}(t)}{\rho_{\text {jam }}}\right]^{l}\right)^{m},\right.
$$

where $h$ is the sample time interval; $t$ denotes the $t$-th time instant; $i \in\left\{1, \cdots, I_{N}\right\}$ is the $i$-th section of a freeway; $I_{N}$ is the total section number; $\tau, v, \kappa, l, m$ are constant parameters reflecting particular characteristics of a given traffic system. The other model parameter variables are listed in Table 1.

\section{Table 1}

Model Parameters

\begin{tabular}{l|l}
\hline Traffic density & $\rho_{i}(t)(\mathrm{veh} / \mathrm{lane} / \mathrm{km})$ \\
\hline Mean speed & $v_{i}(t)(\mathrm{km} / \mathrm{h})$ \\
\hline Traffic flow & $q_{i}(t)(\mathrm{veh} / \mathrm{h})$ \\
\hline Traffic volume On-ramp & $r_{i}(t)(\mathrm{veh} / \mathrm{h})$ \\
\hline Traffic volume Off-ramp & $s_{i}(t)(\mathrm{veh} / \mathrm{h})$ \\
\hline Length & $L_{i}(\mathrm{~km})$ \\
\hline Free speed & $V_{\text {free }}$ \\
\hline Maximum density & $\rho_{\text {jam }}$ \\
\hline
\end{tabular}

Assume the freeway traffic system is repetitive over a finite running time interval $t=\{0,1, \cdots, T\}$. The control objective is to design an adaptive ILC without knowing the exact traffic model and disturbances. The adaptive ILC will make use of the historical freeway traffic data to generate a sequence of control inputs that drives traffic density to converge to the desired one over the entire interval $t=\{0,1, \cdots, T\}$.

\subsection{General Nonlinear Formulation and Some Assumptions}

According to (1) - (4), we can also express the traffic dynamics in a general nonlinear form [17] as

$$
\boldsymbol{y}(t+1)=\boldsymbol{f}[\boldsymbol{y}(t), \boldsymbol{r}(t), \boldsymbol{d}(t)],
$$

where the state vector $\boldsymbol{y}(t) \in R^{n}$ comprises all traffic densities and mean speeds, as well as all ramp queues; the control vector $\boldsymbol{r}(t) \in R^{n}$ comprises all controllable ramp volumes; the disturbance vector $\boldsymbol{d}(t) \in R^{p}$ comprises all on-ramp demands and turning rates; $f(\cdots) \in R^{n}$ is a vector valued function.
Assumption 1: The partial derivatives of $\boldsymbol{f}(\cdots)$ with respect to control inputs $\boldsymbol{r}(t)$ are continuous.

Assumption 2: System (5) is generalized Lipschitz, that is, $\|\Delta \boldsymbol{y}(t+1)\| \leq b_{\Phi}\|\Delta \boldsymbol{r}(t)\|$ for each fixed $t$ and $\|\Delta \boldsymbol{r}(t)\| \neq 0$, where $\Delta \boldsymbol{y}(t+1)=\boldsymbol{y}(t+1)-\boldsymbol{y}(t)$, $\Delta \boldsymbol{r}(t)=\boldsymbol{r}(t)-\boldsymbol{r}(t-1)$, and $b_{\Phi}$ is a positive constant.

Remark 1: Assumption 1 is a typical condition of control system design for general nonlinear systems. It is obvious that the partial derivatives of all the non-linear functions in (1) - (4) with respect to its arguments exist and are continuous. Therefore, Assumption 1 is satisfied for the considered freeway traffic system.

Remark 2: Assumption 2 limits the rates of changes of the system outputs driven by the changes of the control inputs. For a real freeway traffic system, it is evident that the finite change of one traffic variable does not cause an infinite variation of the other variables. Furthermore, we just need the existence of such a constant $b_{\Phi}$ without requiring the exact value.

\subsection{Dynamical Linearization}

The equivalent dynamical linearization is summarized as the following theorem [9].

Theorem 1: For nonlinear system (5), satisfying Assumptions 1 and 2 with $\|\Delta r(t)\| \neq 0$ for each fixed $t$, there must exist a parameter $\boldsymbol{\Phi}(t)$, called the PPD matrix, such that nonlinear system (5) can be transformed into the following equivalent dynamical linearization data model

$$
\Delta y(t+1)=\boldsymbol{\Phi}(t) \Delta \boldsymbol{r}(t),
$$

where $\boldsymbol{\Phi}(t) \in R^{n \times n}$ and $\|\boldsymbol{\Phi}(t)\| \leq b_{\Phi}$.

Proof. See Appendix A.

Remark 2: The linear data-model (6) is an equivalent and exact description of system (5), which is quite different from other linearization forms such as Taylor's linearization, where the high-order terms are omitted. The dynamical linearization is a data-driven approach and it is achieved only based on the I/O data of the plant. Further, the linear data-model is simple without requiring any fuzzy rules as in fuzzy control, or any external testing signals and any training process as in neural network control.

Another assumption is exposed on the PPD parameter. Assumption 3:.The PPD matrix parameter $\boldsymbol{\Phi}(t)$ is 
positive or negative definite. Without loss of generality, we assume $\boldsymbol{\Phi}(t) \geq \delta \boldsymbol{I}>\boldsymbol{0}$ in this paper.

Remark 3: Assumption 3 is similar to the control direction, which is common in control systems.

As shown in [9], $\boldsymbol{\Phi}(t)$ represents the Markov parameters or the transfer function of a linear system. It is reasonable to assume that $\boldsymbol{\Phi}(t)$ is strictly repeatable for the freeway traffic system which varies along time axis only over a finite time interval $t \in\{0,1, \cdots, T\}$. Then, by considering the repeatability of the freeway traffic control system, the equivalent expression of (5) is developed as

$$
\boldsymbol{y}_{k}(t+1)=\boldsymbol{y}_{k}(t)+\boldsymbol{\Phi}(t) \Delta \boldsymbol{r}_{k}(t),
$$

where $\Delta \boldsymbol{r}_{k}(t)=\boldsymbol{r}_{k}(t)-\boldsymbol{r}_{k}(t-1) ; t=\{0,1, \cdots, T\} ; k=1,2, \ldots$ is the iteration number.

\section{Linear Data-Model Based Adaptive ILC}

The desired traffic output is $\boldsymbol{y}_{d, k}(t) \in R^{n}$, which is iteration-dependant and bounded for all $t \in\{0, \cdots, T\}$ and $k=1,2, \cdots$, i.e.,

$\sup _{k}\left\|\boldsymbol{y}_{d, k}(t)\right\| \leq b_{y_{d}}, \forall t \in\{0, \cdots, T\}$

where we only need to know the existence of positive constant $b_{y_{d}}$.

Define the tracking error $\boldsymbol{e}_{k}(t)=\boldsymbol{y}_{d, k}(t)-\boldsymbol{y}_{k}(t)$, $t \in\{0,1, \cdots, T\}$. From (7), one can obtain

$$
\begin{aligned}
\mathbf{e}_{k}(t+1) & =\mathbf{y}_{d, k}(t+1)-\mathbf{y}_{k}(t)-\boldsymbol{\Phi}(t) \Delta \mathbf{r}_{k}(t) \\
& =\boldsymbol{\Phi}(t)\left(\boldsymbol{\Phi}(t)^{-1} \mathbf{y}_{d, k}(t+1)-\boldsymbol{\Phi}(t)^{-1} \mathbf{y}_{k}(t)-\Delta \mathbf{r}_{k}(t)\right)
\end{aligned}
$$

Let $\boldsymbol{\zeta}_{k}(t)=\boldsymbol{y}_{d, k}(t+1)-\boldsymbol{y}_{k}(t) \in R^{n}$ and $\boldsymbol{\Theta}(t)=\boldsymbol{\Phi}(t)^{-1} \in$ $R^{n \times n}$. Equation (8) is rewritten as

$$
\boldsymbol{e}_{k}(t+1)=\boldsymbol{\Phi}(t)\left[\boldsymbol{\Theta}(t) \boldsymbol{\zeta}_{k}(t)-\Delta \boldsymbol{r}_{k}(t)\right] .
$$

Then the learning control law at the $k$-th iteration is proposed as

$$
\boldsymbol{r}_{k}(t)=\boldsymbol{r}_{k}(t-1)+\hat{\boldsymbol{\Theta}}_{k}(t) \boldsymbol{\zeta}_{k}(t), t=\{0,1, \cdots, T-1\}, \quad \text { (10) }
$$

where $\hat{\boldsymbol{\Theta}}_{0}(t)$ is the estimation of $\boldsymbol{\Theta}(t)$ and it is updated iteratively as

$$
\hat{\boldsymbol{\Theta}}_{k}(t)=\hat{\boldsymbol{\Theta}}_{k-1}(t)+\frac{a \boldsymbol{P} \boldsymbol{e}_{k-1}(t+1)}{c+\boldsymbol{\zeta}_{k-1}(t)^{T} \boldsymbol{\zeta}_{k-1}(t)} \boldsymbol{\zeta}_{k-1}(t)^{T},
$$

where $\hat{\boldsymbol{\Theta}}_{0}(t)$ being given bounded; $c>0 ; 0<a b_{\Phi}<2$, with $b_{\Phi}$ being defined in assumption A2; $\boldsymbol{P}=\boldsymbol{I}_{n \times n}$ is an identical matrix.

Remark 4: Different from other adaptive ILC [5-7, 14, $17,22], \boldsymbol{\zeta}_{k}(t)=\boldsymbol{y}_{d, k}(t+1)-\boldsymbol{y}_{k}(t)$ is a linear function about the system output and reference trajectory. Thus, it is automatically satisfied with linear grow condition with respect to the tracking error.

Remark 5: The main differences of the proposed data-driven AILC from the ILC approach proposed in [6] are that: (a) As the basis of the controller design, the dynamic linear model (6) in this paper is built by using time-difference along the time axis. While the dynamic linear model in [6] is built using iteration-difference between two consecutive iterations. (b) The learning control law (10) and the parameter updating law together make up an adaptive mechanism, which the approach in [6] is designed using optimization technique. (c) The desired reference is incorporated in the learning law (10) such that the proposed method in this work can deal with iteration-varying reference trajectory. However, the desired tracking trajectory is required identical for all iterations in [6].

Remark 6: The presented LDM-AILC is a data-driven approach since the controllers design and analysis merely uses the input and output measurement data of the plant. The unknown parameter $\boldsymbol{\Theta}(t)$ is estimated iteratively just by using the measured I/O data of the controlled system.

The convergence results of the proposed LDM-AILC scheme (10) - (11) for MIMO nonlinear discrete-time system (5) are summarized as the following theorem.

Theorem 2: For system (5) under Assumptions 1 - 3, the control law (10) together with the learning updating law (11) guarantees that (a) the PPD matrix estimation $\hat{\boldsymbol{\Theta}}_{k}(t)$ is bounded for all $t \in\{0, \cdots, T\}$ and $k=1,2, \cdots$, and (b) the tracking error $\boldsymbol{e}_{k}(t)$, $t \in\{1, \cdots, T\}$, converges to zero iteratively as $k$ approaches to infinity.

Proof: The proof of this theorem is similar to that in [6], and the detail progress is shown in Appendix B for the convenience of readers. 


\section{Simulation Study}

For the simulation, we consider a long segment of freeway that is subdivided into 12 sections. The length of each section is $0.5 \mathrm{~km}$. The initial traffic volume entering section 1 is 1600 vehicles per hour. The parameters used in this model are given as: $v_{i}(0)=50 \mathrm{~km} / \mathrm{h}$, $v_{\text {free }}=80 \mathrm{~km} / \mathrm{h}, \rho_{\text {jam }}=80 \mathrm{veh} /$ lane $/ \mathrm{km}, l=1.8, \quad m=1.7$, $\kappa=13 \mathrm{veh} / \mathrm{km}, \quad \tau=0.01 \mathrm{~h}, \quad h=0.00417 \mathrm{~h}, \quad \gamma=35 \mathrm{~km}^{2} / \mathrm{h}$, $r_{i}(0)=0 v e h / h, \alpha=0.95$.

There are one on-ramp located in Section 2 with known traffic demand and two off-ramps located in Section 5 and Section 9 with unknown exiting traffic flow, respectively. They were chosen to simulate a traffic scenario during rush hour. The unknown existing flows actually are chosen to mimic the exogenous disturbances to Section 2.

Note that the queuing demands actually impose certain constraints on the control inputs of ramp metering, i.e., the on-ramp volumes cannot exceed the current demands plus the existing waiting queues at on-ramps at the time $k$; thus

$$
r_{i}(t) \leq \eta_{i}(t)+\frac{l_{i}(t)}{T}, \quad i \in I_{O N},
$$

where $l_{i}(t)$ denotes the length (in vehicles) of a possibly existing waiting queue at time instant $t$ at the $i$-th on-ramp; $\eta_{i}(t)$ is the traffic demand flow at time instant $t$ at the $i$-th on-ramp (veh/h), and $I_{O N}=2$ for the simulation of this paper, denotes the set of indices of the sections where an on-ramp exists. On the other hand, the waiting queue is the accumulation of the difference between the demand and actual on-ramp, i.e.,

$$
l_{i}(t+1)=l_{i}(t)+T\left[\eta_{i}(t)-r_{i}(t)\right] \quad i \in I_{O N} .
$$

The desired freeway traffic density is $\rho_{d, k}=30+0.1 \sin (\pi k / 50)$, which is iteration-varying as shown in Fig. 2. The random initial traffic density is selected as $\rho_{i, k}(0)=30+0.10$ rand, shown in Fig. 3 .

In the simulation, we choose $a=0.1, c=0.01$, $\theta_{0}(t)=0.002, \quad u_{0}(t)=0$. Applying the proposed LDM-AILC, the learning convergence is shown in Fig. 4 where the horizon is the iteration number and the vertical axis is the maximum absolute value of tracking error $e_{\max , k}^{*}=\max _{t \in\{1, \cdots, 500\}}\left\{\left|e_{k}(t)\right|\right\}$.
Figure 2

The profile of iteration-variant desired traffic density

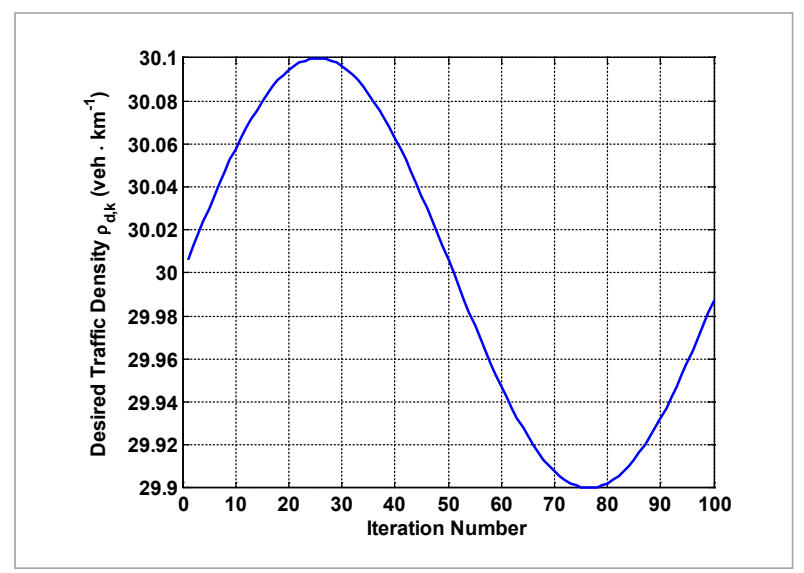

Figure 3

The profile of initial traffic density over 100 iterations

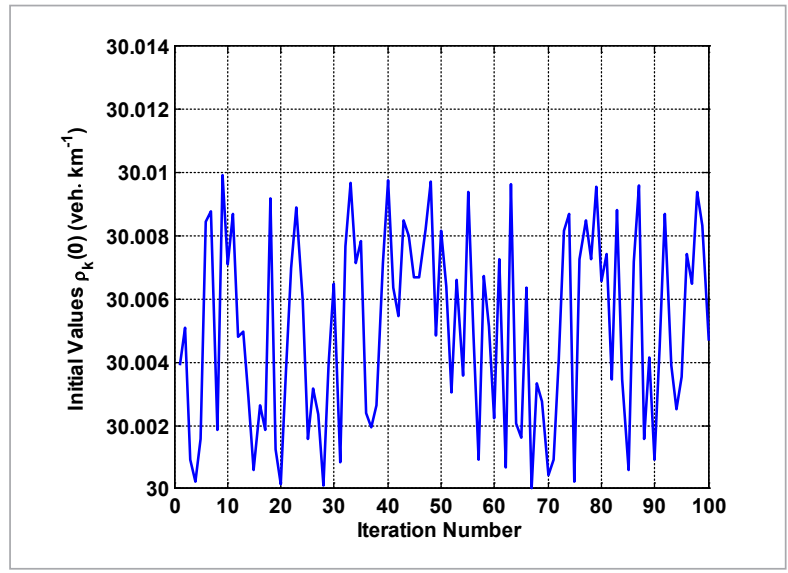

Figure 4

The profile of maximum tracking error in the time interval of $t \in\{0, \cdots, 500\}$

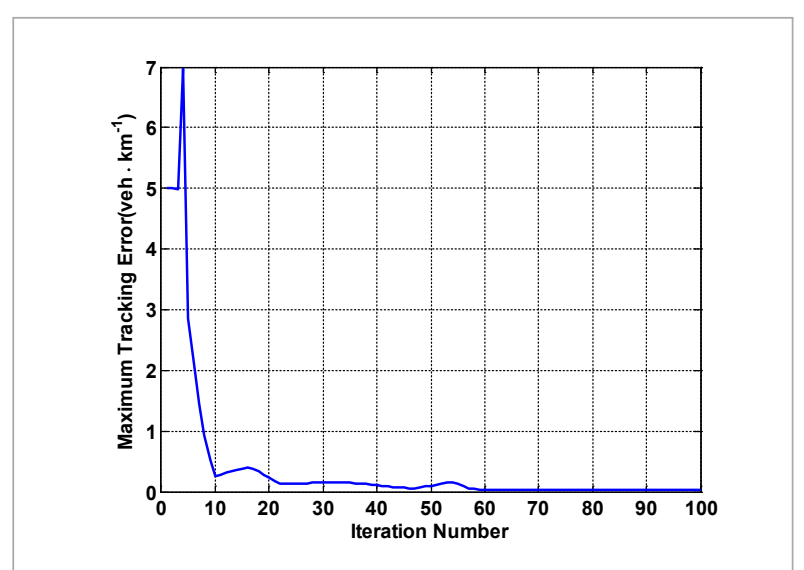


The effectiveness of the proposed LDM-AILC can be seen from Figures 2-4. Despite the random initial values and the random variations of the reference trajectory along the iteration axis, the tracking error still approaches to zero asymptotically.

\section{Conclusion}

In this paper, a new linear data-model based adaptive ILC is proposed to solve the local on-ramp control problem at the macroscopic level in a freeway environment by considering non-strictly repeatable conditions. The controller design and analysis only

\section{Appendices}

\section{Appendix A}

\section{Proof of Theorem 1}

Proof: From system (5), we have

$$
\begin{aligned}
\Delta \mathbf{y}(t+1)= & \mathbf{f}[\mathbf{y}(t), \mathbf{r}(t), \mathbf{d}(t)]-\mathbf{f}[\mathbf{y}(t-1), \mathbf{r}(t-1), \mathbf{d}(t-1)] \\
& =\mathbf{f}[\mathbf{y}(t), \mathbf{r}(t), \mathbf{d}(t)]-\mathbf{f}[\mathbf{y}(t), \mathbf{r}(t-1), \mathbf{d}(t)] \\
& +\mathbf{f}[\mathbf{y}(t), \mathbf{r}(t-1), \mathbf{d}(t)]-\mathbf{f}[\mathbf{y}(t-1), \mathbf{r}(t-1), \mathbf{d}(t-1)] .
\end{aligned}
$$

Let $\boldsymbol{\Psi}(t)=\boldsymbol{f}[\boldsymbol{y}(t), \boldsymbol{r}(t-1), \boldsymbol{d}(t)]-\boldsymbol{f}[\boldsymbol{y}(t-1), \boldsymbol{r}(t-1), \boldsymbol{d}(t-1)]$. By virtue of Assumption 1 and the differential mean value theorem, (A1) can be rewritten as

$$
\Delta \boldsymbol{y}(t+1)=\frac{\partial \boldsymbol{f}^{*}}{\partial \boldsymbol{r}(t)} \Delta \boldsymbol{r}(t)+\boldsymbol{\Psi}(t),
$$

where

$$
\frac{\partial \boldsymbol{f}^{*}}{\partial \boldsymbol{r}(t)}=\left[\begin{array}{cccc}
\frac{\partial f_{1}^{*}}{\partial r_{1}(t)} & \frac{\partial f_{1}^{*}}{\partial r_{2}(t)} & \cdots & \frac{\partial f_{1}^{*}}{\partial r_{n}(t)} \\
\frac{\partial f_{2}^{*}}{\partial r_{1}(t)} & \frac{\partial f_{2}^{*}}{\partial r_{2}(t)} & \cdots & \frac{\partial f_{2}^{*}}{\partial r_{n}(t)} \\
\vdots & \vdots & \vdots & \vdots \\
\frac{\partial f_{n}^{*}}{\partial r_{1}(t)} & \frac{\partial f_{n}^{*}}{\partial r_{2}(t)} & \cdots & \frac{\partial f_{n}^{*}}{\partial r_{n}(t)}
\end{array}\right] \in R^{n \times n},
$$

$\partial f_{i}^{*} / \partial r_{j}(t)$ represents the partial derivative value of $f_{i}$ with respect to input $r_{j}(t)$ at some point in the interval $\left[r_{j}(t), r_{j}(t-1)\right]$. depends on the measured I/O data of the freeway traffic system without requiring any other model information. Rigorous mathematical analysis is provided to show the asymptotic convergence of the proposed LDM-AILC without assuming any identical conditions on both the initial state and the reference trajectory. Simulation results show the effectiveness of the proposed LDM-AILC based traffic control scheme.

\section{Acknowledgements}

This work was supported by National Nature Science Foundation of China (61374102), and the Taishan Scholar program of Shandong Province.

For each fixed $t$, we consider the following equation with numerical matrix $\boldsymbol{H}(t)$ with $\mathrm{n}$ rows and $\mathrm{n}$ columns:

$$
\boldsymbol{\Psi}(t)=\boldsymbol{H}(t) \Delta \boldsymbol{r}(t) .
$$

Since condition $\|\Delta r(t)\| \neq 0$ holds, Equation (A3) must have at least one solution $\boldsymbol{H}^{*}(t)$. In fact, it must have infinite number of solutions for each $t$.

Let $\boldsymbol{\Phi}(t)=\boldsymbol{H}^{*}(t)+\left(\partial \boldsymbol{f}^{*} / \partial \boldsymbol{r}(t)\right)$, then we have $\Delta \boldsymbol{x}(t+1)=\boldsymbol{\Phi}(t) \Delta \boldsymbol{r}(t)$. Result $\|\boldsymbol{\Phi}(t)\| \leq b_{\Phi}$ is the direct conclusion of Assumption 2.

\section{Appendix B.}

\section{Proof of Theorem 2}

Define the parametric estimation error $\widetilde{\boldsymbol{\Theta}}_{k}=\boldsymbol{\Theta}(t)-\hat{\boldsymbol{\Theta}}_{k}(t)$. Substituting the control law (10) into the error dynamics (9) yields

$$
\begin{aligned}
\mathbf{e}_{k}(t+1) & =\boldsymbol{\Phi}(t)\left[\boldsymbol{\Theta}(t) \boldsymbol{\zeta}_{k}(t)-\hat{\boldsymbol{\Theta}}_{k}(t) \boldsymbol{\zeta}_{k}(t)\right] \\
& =\boldsymbol{\Phi}(t) \tilde{\boldsymbol{\Theta}}_{k}(t) \boldsymbol{\zeta}_{k}(t) .
\end{aligned}
$$

Note that the following properties of trace hold in general

P1.

$$
\begin{aligned}
& \operatorname{trace}\left[(\mathbf{A}-\mathbf{B})^{T}(\mathbf{A}-\mathbf{B})\right]-\operatorname{trace}\left[(\mathbf{C}-\mathbf{B})^{T}(\mathbf{C}-\mathbf{B})\right] \\
& \quad=\operatorname{trace}\left[(\mathbf{A}-\mathbf{C})^{T}(\mathbf{A}+\mathbf{C}-2 \mathbf{B})\right] .
\end{aligned}
$$


P2.

$\operatorname{trace}\left(\boldsymbol{Q}^{T} \boldsymbol{v} \boldsymbol{y}^{T}\right)=\operatorname{trace}\left(\boldsymbol{Q}^{T} \boldsymbol{v} \boldsymbol{y}^{T}\right)^{T}=\boldsymbol{v}^{T} \boldsymbol{Q} \boldsymbol{y}$

where $A, B$, and $\boldsymbol{C}$ are square matrices, $\boldsymbol{Q} \in R^{m \times n}$, $\boldsymbol{v} \in R^{m \times 1}$, and $\boldsymbol{y} \in R^{n \times 1}$.

Define $\quad V_{k}(t)=\operatorname{trace}\left[\left(\hat{\boldsymbol{\Theta}}_{k}(t)-\boldsymbol{\Theta}(t)\right)^{T}\left(\hat{\boldsymbol{\Theta}}_{k}(t)-\boldsymbol{\Theta}(t)\right)\right]$, then according to the above trace property $\mathrm{P} 1$, it is derived that

$$
\begin{aligned}
\Delta V_{k}(t) & =V_{k}(t)-V_{k-1}(t) \\
& =\operatorname{trace}\left[\left(\hat{\boldsymbol{\Theta}}_{k}(t)-\boldsymbol{\Theta}(t)\right)^{T}\left(\hat{\boldsymbol{\Theta}}_{k}(t)-\boldsymbol{\Theta}(t)\right)\right] \\
& -\operatorname{trace}\left[\left(\hat{\boldsymbol{\Theta}}_{k-1}(t)-\boldsymbol{\Theta}(t)\right)^{T}\left(\hat{\boldsymbol{\Theta}}_{k-1}(t)-\boldsymbol{\Theta}(t)\right)\right] \\
& =\operatorname{trace}\left[\left(\hat{\boldsymbol{\Theta}}_{k}(t)-\hat{\boldsymbol{\Theta}}_{k-1}(t)\right)^{T}\left(\hat{\boldsymbol{\Theta}}_{k}(t)+\hat{\boldsymbol{\Theta}}_{k-1}(t)-2 \boldsymbol{\Theta}(t)\right)\right] .
\end{aligned}
$$

According to (11), Eq. (B2) becomes

$$
\begin{aligned}
\Delta V_{k}(t) & =\operatorname{trace}\left[\left(\frac{a \mathbf{P e}_{k-1}(t+1) \zeta_{k-1}(t)^{T}}{c+\zeta_{k-1}(t)^{T} \zeta_{k-1}(t)}\right)^{T}\left(\hat{\boldsymbol{\Theta}}_{k}(t)+\hat{\boldsymbol{\Theta}}_{k-1}(t)-2 \boldsymbol{\Theta}(t)\right)\right. \\
& =\frac{a}{c+\zeta_{k-1}(t)^{T} \zeta_{k-1}(t)} \\
& \operatorname{trace}\left[\left(\mathbf{P e}_{k-1}(t+1) \zeta_{k-1}(t)^{T}\right)^{T}\left(\hat{\boldsymbol{\Theta}}_{k}(t)+\hat{\boldsymbol{\Theta}}_{k-1}(t)-2 \boldsymbol{\Theta}(t)\right)\right] .
\end{aligned}
$$

(B3)

In terms of the above trace property P2, (B3) yields

$$
\begin{aligned}
\Delta V_{k}(t) & =\frac{a}{c+\boldsymbol{\zeta}_{k-1}(t)^{T} \boldsymbol{\zeta}_{k-1}(t)} \\
& \operatorname{trace}\left[\left(\hat{\boldsymbol{\Theta}}_{k}(t)+\hat{\boldsymbol{\Theta}}_{k-1}(t)-2 \boldsymbol{\Theta}(t)\right)^{T}\left(\mathbf{P} \mathbf{e}_{k-1}(t+1) \boldsymbol{\zeta}_{k-1}(t)^{T}\right)\right] \\
& =\frac{a\left(\mathbf{P e}_{k-1}(t+1)\right)^{T}\left(\hat{\boldsymbol{\Theta}}_{k}(t)+\hat{\boldsymbol{\Theta}}_{k-1}(t)-2 \boldsymbol{\Theta}(t)\right) \zeta_{k-1}(t)}{c+\zeta_{k-1}(t)^{T} \zeta_{k-1}(t)} \\
& =\frac{a \mathbf{e}_{k-1}(t+1)^{T} \mathbf{P}\left(\hat{\boldsymbol{\Theta}}_{k}(t)+\hat{\boldsymbol{\Theta}}_{k-1}(t)-2 \boldsymbol{\Theta}(t)\right) \zeta_{k-1}(t)}{c+\zeta_{k-1}(t)^{T} \zeta_{k-1}(t)} .
\end{aligned}
$$

(B4)

Subtracting $\boldsymbol{\Theta}(t)$ from both sides of (11) and using relationship (B1), one obtains

$$
\begin{aligned}
\tilde{\boldsymbol{\Theta}}_{k}(t) & =\tilde{\boldsymbol{\Theta}}_{k-1}(t)-\frac{a \mathbf{P} \mathbf{e}_{k-1}(t+1)}{c+\boldsymbol{\zeta}_{k-1}(t)^{T} \zeta_{k-1}(t)} \zeta_{k-1}(t)^{T} \\
& =\tilde{\boldsymbol{\Theta}}_{k-1}(t)-\frac{a \mathbf{P} \boldsymbol{\Phi}(t) \zeta_{k-1}(t)^{T} \zeta_{k-1}(t)}{c+\zeta_{k-1}(t)^{T} \zeta_{k-1}(t)} \tilde{\boldsymbol{\Theta}}_{k-1}(t) \\
& =\left[\mathbf{I}-\frac{a \mathbf{P} 5)}{c+(t) \zeta_{k-1}(t)^{T} \zeta_{k-1}(t)}\right] \tilde{\boldsymbol{\Theta}}_{k-1}(t)^{T} \zeta_{k-1}(t)
\end{aligned}
$$

Hence in terms of (B5), one can rearrange (B4) as

$$
\begin{aligned}
\Delta V_{k}(t) & =\frac{a \mathbf{e}_{k-1}(t+1)^{T} \mathbf{P}}{c+\zeta_{k-1}(t)^{T} \zeta_{k-1}(t)} \\
& \left(\hat{\boldsymbol{\Theta}}_{k}(t)-\boldsymbol{\Theta}(t)+\hat{\boldsymbol{\Theta}}_{k-1}(t)-\boldsymbol{\Theta}(t)\right) \zeta_{k-1}(t) \\
& =\frac{a \mathbf{e}_{k-1}(t+1)^{T} \mathbf{P}}{c+\zeta_{k-1}(t)^{T} \zeta_{k-1}(t)}\left(-\tilde{\boldsymbol{\Theta}}_{k}(t)-\tilde{\boldsymbol{\Theta}}_{k-1}(t)\right) \zeta_{k-1}(t) \\
& =\frac{a \mathbf{e}_{k-1}(t+1)^{T} \mathbf{P}}{c+\zeta_{k-1}(t)^{T} \zeta_{k-1}(t)} \\
& {\left[-2 \mathbf{I}+\frac{a \mathbf{P} \boldsymbol{\Phi}(t) \zeta_{k-1}(t)^{T} \zeta_{k-1}(t)}{c+\zeta_{k-1}(t)^{T} \zeta_{k-1}(t)}\right] \tilde{\boldsymbol{\Theta}}_{k-1}(t) \zeta_{k-1}(t) }
\end{aligned}
$$

Again using (B1), we have

$$
\begin{aligned}
\Delta V_{k}(t) & =\frac{a \mathbf{e}_{k-1}(t+1)^{T} \mathbf{P}}{c+\zeta_{k-1}(t)^{T} \zeta_{k-1}(t)}\left[-2 \mathbf{I}+\frac{a \mathbf{P} \boldsymbol{\Phi}(t) \zeta_{k-1}(t)^{T} \zeta_{k-1}(t)}{c+\zeta_{k-1}(t)^{T} \zeta_{k-1}(t)}\right] \\
& {[\mathbf{P} \boldsymbol{\Phi}(t)]^{-1}[\mathbf{P} \boldsymbol{\Phi}(t)] \tilde{\boldsymbol{\Theta}}_{k-1}(t) \zeta_{k-1}(t) } \\
& =\frac{a[\mathbf{P} \boldsymbol{\Phi}(t)]^{-1} \mathbf{e}_{k-1}(t+1)^{T} \mathbf{P}}{c+\zeta_{k-1}(t)^{T} \zeta_{k-1}(t)} \\
& {\left[-2 \mathbf{I}+\frac{a \mathbf{P} \boldsymbol{\Phi}(t) \zeta_{k-1}(t)^{T} \zeta_{k-1}(t)}{c+\zeta_{k-1}(t)^{T} \boldsymbol{\zeta}_{k-1}(t)}\right] \mathbf{P e}_{k-1}(t+1) } \\
& =a[\mathbf{P} \boldsymbol{\Phi}(t)]^{-1}\left[-2 \mathbf{I}+\frac{a \mathbf{P} \boldsymbol{\Phi}(t) \zeta_{k-1}(t)^{T} \zeta_{k-1}(t)}{c+\zeta_{k-1}(t)^{T} \zeta_{k-1}(t)}\right] \\
& \frac{\mathbf{e}_{k-1}(t+1)^{T} \mathbf{P}^{2} \mathbf{e}_{k-1}(t+1)}{c+\boldsymbol{\zeta}_{k-1}(t)^{T} \zeta_{k-1}(t)}
\end{aligned}
$$

Since $0<a b_{\Phi}<2, q>0$, it is obvious

$$
a[\mathbf{P} \Phi(t)]^{-1}\left[-2 \mathbf{I}+\frac{a \mathbf{P} \boldsymbol{\Phi}(t) \zeta_{k-1}(t)^{T} \zeta_{k-1}(t)}{c+\zeta_{k-1}(t)^{T} \zeta_{k-1}(t)}\right]<0 .
$$


As a result of (B7) and (B8), it is easy to get

$$
\begin{aligned}
& \operatorname{trace}\left[\left(\hat{\boldsymbol{\Theta}}_{k}(t)-\boldsymbol{\Theta}(t)\right)^{T}\left(\hat{\boldsymbol{\Theta}}_{k}(t)-\boldsymbol{\Theta}(t)\right)\right] \\
& \leq \operatorname{trace}\left[\left(\hat{\boldsymbol{\Theta}}_{k-1}(t)-\boldsymbol{\Theta}(t)\right)^{T}\left(\hat{\boldsymbol{\Theta}}_{k-1}(t)-\boldsymbol{\Theta}(t)\right)\right] \\
& \leq \cdots \leq \operatorname{trace}\left[\left(\hat{\boldsymbol{\Theta}}_{0}(t)-\boldsymbol{\Theta}(t)\right)^{T}\left(\hat{\boldsymbol{\Theta}}_{0}(t)-\boldsymbol{\Theta}(t)\right)\right]
\end{aligned}
$$

or

$$
\left\|\hat{\boldsymbol{\Theta}}_{k}(t)-\boldsymbol{\Theta}(t)\right\| \leq\left\|\hat{\boldsymbol{\Theta}}_{k-1}(t)-\boldsymbol{\Theta}(t)\right\| \leq \cdots \leq\left\|\hat{\boldsymbol{\Theta}}_{0}(t)-\boldsymbol{\Theta}(t)\right\|
$$

According to Theorem 1 and Assumption 3, $0<\delta \leq\|\boldsymbol{\Phi}(t)\| \leq b_{\Phi}$, so $\boldsymbol{\Theta}(t)$ is bounded. Further, $\hat{\boldsymbol{\Theta}}_{0}(t), t \in\{0, \cdots, T\}$ is given bounded too, thus it is obvious that $\left\|\hat{\boldsymbol{\Theta}}_{0}(t)-\boldsymbol{\Theta}(t)\right\|$ is bounded. Therefore, inequality (B10) implies that $\left\|\widetilde{\boldsymbol{\Theta}}_{k}(t)\right\|$ is nonnegative, nonincreasing, and bounded and $\hat{\boldsymbol{\Theta}}_{k}(t)$ is bounded.

Summing both sides of (Br) from 0 to $k$, yields

$$
\begin{aligned}
& V_{k}(t)=V_{0}(t)+a[\mathbf{P} \boldsymbol{\Phi}(t)]^{-1} \\
& * \sum_{j=1}^{k}\left[-2 \mathbf{I}+\frac{a \mathbf{P} \boldsymbol{\Phi}(t) \zeta_{j-1}(t)^{T} \zeta_{j-1}(t)}{c+\zeta_{j-1}(t)^{T} \zeta_{j-1}(t)}\right] \frac{\mathbf{e}_{j-1}(t+1)^{T} \mathbf{P}^{2} \mathbf{e}_{j-1}(t+1)}{c+\zeta_{j-1}(t)^{T} \zeta_{j-1}(t)} .
\end{aligned}
$$

\section{References}

1. Chang, T. H., Li, Z. Y. Optimization of Mainline Traffic via an Adaptive Coordinated Ramp-Metering Control Model with Dynamic OD Esti-mation. Transportation Research, Part C, 2002, 10(2), 99-120.https://doi. org/10.1016/S0968-090X(01)00006-7

2. Chien,C. J. A Combined Adaptive Law for Fuzzy Iterative Learning Control of Nonlinear Sys-tems with Varying Control Tasks. IEEE Transac-tions on Fuzzy Systems, 2008, 16(1), 40-51. https://doi.org/10.1109/ TFUZZ.2007.902021

3. Chien, C. J., Fu, L. C. An Iterative Learning Control of Nonlinear Systems Using Neural Net-work Design. Asian Journal of Control, 2002, 4(1), 21-29. https://doi. org/10.1111/j.1934-6093.2002.tb00329.x

4. Chien, C. J., Yao, C.Y.An Output-Based Adap-tive Iterative Learning Controller for High Relative Degree Uncertain Linear Systems. Automatica, 2004, 40(1), 145-153. https://doi.org/10.1016/j.automatica.2003.09.002
Since $V_{0}(t)$ is bounded and $V_{k}(t)$ is nonnegative and bounded, in terms of (B8) and (B11), one obtains

$$
\lim _{k \rightarrow \infty} \frac{\left\|\boldsymbol{e}_{k}(t+1)\right\|^{2}}{c+\boldsymbol{\zeta}_{k}(t)^{T} \zeta_{k}(t)}=0 .
$$

According to the definition of $\hat{\boldsymbol{\Theta}}_{k}(t)$, one has

$$
\begin{aligned}
\left\|\boldsymbol{\zeta}_{k}(t)\right\| & \leq\left\|\mathbf{y}_{d, k}(t+1)\right\|+\left\|\mathbf{y}_{k}(t)\right\| \\
& \leq\left\|\mathbf{y}_{d, k}(t+1)\right\|+\left\|\mathbf{y}_{d, k}(t)\right\|+\left\|\mathbf{e}_{k}(t)\right\| \\
& \leq\left\|\mathbf{y}_{d, k}(t+1)\right\|+\left\|\mathbf{y}_{d, k}(t)\right\|+\max _{i \in\{0, \cdots, t\}}\left\|\mathbf{e}_{k}(i+1)\right\| \\
& \leq q_{1}+q_{2} \max _{i \in\{0, \cdots, t\}}\left\|\mathbf{e}_{k}(i+1)\right\|,
\end{aligned}
$$

where $q_{1}=2 b_{y_{d}}$ and $q_{2}=1$ are two positive constants. Thus, the convergence property (B12) together with (B13) implies the asymptotical convergence of $\boldsymbol{e}_{k}(t)$ along iteration axis for all $t \in\{1, \cdots, T\}$.

5. Chi, R. H., Hou, Z. S. A New Neural Network-Based Adaptive ILC for Nonlinear Discrete-time Systems with Dead Zone Scheme. Journal of Sys-tems Science and Complexity, 2009, 22(3), 435-445. https://doi. org/10.1007/s11424-009-9176-4

6. Chi, R. H., Hou, Z. S. Dual Stage Optimal Itera-tive Learning Control for Nonlinear Non-affine Discrete-time Systems. ACTA AUTOMATICA SINICA, 2007, 33(10), 1061-1065. https://doi.org/10.1360/aas-007-1061

7. Chi, R. H., Hou, Z. S., Xu, J. X. Adaptive ILC for a Class of Discrete-time Systems with Iteration-varying Trajectory and Random Initial Condition. Automatica, 2008, 44, 2207-2213. https://doi.org/10.1016/j.automatica.2007.12.004

8. Doh, T. Y., Ryoo, J. R., Chang, D. E. Robust It-erative Learning Controller Design using the Per-formance Weighting Function of Feedback Control Systems. International Journal of Control, Automa-tion, and Systems, 
2014, 12(1), 63-70. https://doi.org/10.1007/s12555-0129401-0

9. Hou, Z.S., Jin, S.T. Data Driven Model-free Adaptive Control for a Class of MIMO Nonlinear Discrete-Time Systems. IEEE Transactions on Neu-ral Networks, 2011, 22(12), 2173-2188. https://doi.org/10.1109/ TNN.2011.2176141

10. Hou, Z. S., Xu, J. X., Yan, J. W. An Iterative Learning Approach for Density Control of Freeway Traffic Flow via Ramp Metering. Transportation Research, Part C, 2008 16(1), 71-97. https://doi.org/10.1016/j.trc.2007.06.007

11. Hou, Z. S., Xu, J. X., Zhong, H. W. Freeway Traffic Control Using Iterative Learning Control-Based Ramp Metering and Speed Signaling. IEEE Transactions on Vehicular Technology, 2007, 56(2), 466-477. https://doi. org/10.1109/TVT.2007.891431

12. Hou, Z. S., Xu, X., Yan, J. W., Xu, J. X., Xiong, G. A Complementary Modularized Ramp Metering Approach Based on Iterative Learning Control and ALINEA. IEEE Transactions on Intel-ligent Transportation Systems, 2011, 12(4), 1305-1318. https://doi.org/10.1109/ TITS.2011.2157969

13. Ji, H. B. Freeway Traffic Systems: Prediction and Control. In Proceedings of IEEE 46th Vehicu-lar Technology Conference Mobile Technology for the Human Race, 3, 1815-1819.

14. Jin, S. T., Hou, Z. S., Chi, R. H., Liu, X. B. Da-ta-driven Model-free Adaptive Iterative Learning control for a Class of Discrete-Time Nonlinear Sys-tems. Control Theory and Applications, 2012, 29(8), 1001-1009.

15. Kotsialos, A., Papageorgiou, M., Mangeas, M., Haj-Salem, H. Coordinated and Integrated Control of Motorway Networks via Non-linear Optimal Control. Transportation Research, Part C, 2002, 10(1), 65-84. https:// doi.org/10.1016/S0968-090X(01)00005-5

16. Madady, A. An Extended PID Type Iterative Learning Control. International Journal of Control, Automation, and Systems, 2013, 11(3), 470-481. https://doi. org/10.1007/s12555-012-0350-4

17. Papageorgiou, M., Kotsialos, A. Freeway Ramp Metering: An Overview. IEEE Transactions on In-telligent Transportation Systems, 2002, 3(4), 271-281. https:// doi.org/10.1109/TITS.2002.806803

18. Rotariu, I., Steinbuch, M., Ellenbroek, R. Adaptive Iterative Learning Control for High Pre-cision Motion Systems. IEEE Transactions on Con-trol Systems Technology, 2008, 16(5), 1075-1082. https://doi.org/10.1109/ TCST.2007.906319
19. Seo, W. G., Park, B. H., Lee, J. S. Adaptive Fuzzy Learning Control for a Class of Nonlinear Dynamic Systems. International Journal of Intelli-gent Systems, 2000, 15, 1157-1175. https://doi.org/10.1002/1098-111X(200012) 15:12<1157::AID-INT3>3.0.CO;2-V

20. Smaragdis, E., Papageorgiou, M., Kosmatopou-los, E. A Flow Maximizing Adaptive Local Ramp Metering Strategy. Transportation Research, Part B, 2004, 38, 251270. https://doi.org/10.1016/S0191-2615(03)00012-2

21. Wang, Y. C., Chien, C. J. Design and Analysis of Fuzzy-Neural Discrete Adaptive Iterative Learn-ing Control for Nonlinear Plants. International Journal of Fuzzy Systems, 2013, 15(2), 149-158.

22. Xiao, T. F., Li, X. D., Ho, J. K. L. An Adap-tive Discrete-Time ILC Strategy using Fuzzy Sys-tems for Iteration-Varying Reference Trajectory Tracking. International Journal of Control, Auto-mation, and Systems, 2015, 13(1), 222-230. https://doi.org/10.1007/s12555013-0474-1

23. Xu, J. X., Tan, Y. A Composite Energy Func-tion-Based Learning Control Approach for Nonlin-ear Systems with Time-Varying Parametric Uncer-tainties. IEEE Transactions on Automatic Control, 2002, 47(11), 1940-1945. https://doi.org/10.1109/TAC.2002.804460

24. Xu, J. X., Xu, J. On Iterative Learning from Different Tracking Tasks in the Presence of Time-varying uncertainties. IEEE Transactions on Sys-tems, Man, and Cybernetics, 2004, 34(1), 589-597. https://doi. org/10.1109/TSMCB.2003.818433

25. Xu, J. X., Zhao, X. J., Srinivasan, D. Flow Based Local Ramp Metering Using Iterative Learn-ing and PARAMICS Platform. 2009 IEEE Interna-tional Conference on Control and Automation, Christchurch, New Zealand, December 9-11, 2009, 1140-1145. https://doi. org/10.1109/ICCA.2009.5410173

26. Zhang, H. M., Recker, W. W. On Optimal Freeway Ramp Control Policies for Congested Traffic Corridors. Transportation Research Part B, 1999, 33, 417-436. https:// doi.org/10.1016/S0191-2615(98)00045-9

27. Zhang, H. M., Ritchie, S. G., Jayakrishnan, R. Coordinated Traffic Responsive Ramp Control via Nonlinear State Feedback. Transportation Research, Part C, 2001, 9(5), 333-352. https://doi.org/10.1016/S0968090X(00)00044-9

28. Zhao, X., Srinivasan, D., Xu, J. X. Iterative Learning Control of Freeway Flow via Ramp Me-tering and Simulation on PARAMICS, 2009 TENCON, 1-6. https:// doi.org/10.1109/TENCON.2009.5395809 